\title{
Comparison of Amyloid Positivity Rate and Accumulation Pattern between Amnestic and Non-Amnestic Type Mild Cognitive Impairment
}

\author{
Sun Hyung Lee ${ }^{1}$, Jun Ho Lee ${ }^{2}$, Min Soo Byun ${ }^{3}$, Dahyun $\mathrm{Yi}^{4}$, \\ Gijung Jung ${ }^{4,5}$, Jee Eun Park ${ }^{1,6}$, and Dong Young Lee ${ }^{1,4,6} \bowtie$ \\ ${ }^{1}$ Department of Neuropsychiatry, Seoul National University Hospital, Seoul, Republic of Korea \\ ${ }^{2}$ Department of Neuropsychiatry, National Center for Mental Health, Seoul, Republic of Korea \\ ${ }^{3}$ Department of Neuropsychiatry, Seoul National University Bundang Hospital, Seongnam, Republic of Korea \\ ${ }^{4}$ Institute of Human Behavioral Medicine, Medical Research Center, Seoul National University, Seoul, Republic of Korea \\ ${ }^{5}$ Department of Nursing, Graduate School, Kyung Hee University, Seoul, Republic of Korea \\ ${ }^{6}$ Department of Psychiatry, Seoul National University College of Medicine, Seoul, Republic of Korea
}

Objective We aimed to compare cerebral beta-amyloid protein $(\mathrm{A} \beta)$ positivity rate and amyloid accumulation pattern on amyloid positron emission tomography (PET) between mild cognitive impairment (MCI) subtypes, i.e. amnestic mild cognitive impairment (aMCI) and non-amnestic mild cognitive impairment (naMCI).

Methods The study participants were 34 naMCI patients and age-, sex- and education-matched 68 aMCI patients (1:2 ratio) who visited the Dementia and Age-Associated Cognitive Decline Clinic of the Seoul National University Hospital. All participants received comprehensive clinical and neuropsychological assessments and $\left[{ }^{18} \mathrm{~F}\right]$ florbetaben PET.

Results $\mathrm{A} \beta$ positivity rate of naMCI group (26.5\%) was significantly lower than that of aMCI group (64.7\%). Among A $\beta$ positive individuals, there was no difference in $\mathrm{A} \beta$ accumulation pattern between naMCI and aMCI.

Conclusion The findings suggest that MCI subtypes based on impaired cognitive domains have a differential association with brain $A \beta$ deposition, a core pathology of AD. Amnestic subtype of MCI are more closely associated with cerebral A $\beta$ deposition compared to nonamnestic subtype. In contrast, the pattern of amyloid deposition does not appear to have any difference between the subtypes.

Psychiatry Investig 2020;17(6):603-607

Key Words Non-amnestic MCI, Amnestic MCI, Amyloid PET, Amyloid positivity rate, Amyloid accumulation pattern.

\section{INTRODUCTION}

Mild cognitive impairment (MCI) is a clinical condition between cognitively normal aging and dementia. ${ }^{1}$ Based on the presence of memory impairment, MCI is classified into two subtypes, i.e., amnestic MCI (aMCI) and non-amnestic MCI

\footnotetext{
Received: February 17, 2020 Accepted: April 3, 2020

$\triangle$ Correspondence: Dong Young Lee, $\mathrm{MD}, \mathrm{PhD}$

Department of Neuropsychiatry, Seoul National University Hospital, 101 Daehak-ro, Jongno-gu, Seoul 03080, Republic of Korea

Tel: +82-2-2072-2205, Fax: +82-2-744-7241, E-mail: selfpsy@snu.ac.kr

$\triangle$ Correspondence: Jun Ho Lee, MD

Department of Neuropsychiatry, National Center for Mental Health, 127 Yongmasan-ro, Gwangjin-gu, Seoul 04933, Republic of Korea

Tel: +82-2-2204-0162, Fax: +82-2-2204-0381, E-mail: kukulolv@naver.com

(c) This is an Open Access article distributed under the terms of the Creative Commons Attribution Non-Commercial License (https://creativecommons.org/licenses/bync/4.0) which permits unrestricted non-commercial use, distribution, and reproduction in any medium, provided the original work is properly cited.
}

(naMCI). ${ }^{2,3}$ It has been known that the two subtypes of MCI have different clinical characteristics. ${ }^{4,5}$ In particular, individuals with aMCI are more likely to progress to Alzheimer's disease $(\mathrm{AD})$ dementia compared to those with naMCI. ${ }^{6-8}$

In regard of in vivo neuropathological substrates underlying each MCI subtypes, however, sufficient information is still not available. Although several amyloid PET studies indicated that the positivity rate of beta-amyloid protein $(\mathrm{A} \beta)$ deposition, a core neuropathology of $\mathrm{AD}$, is higher in aMCI than in naMCI, ${ }^{9-12}$ the sample sizes of naMCI subjects included in previous studies were too small (only 7-18) to make any conclusion. In addition to $A \beta$ positivity rate, one previous study ${ }^{11}$ investigated the patterns of cerebral $\mathrm{A} \beta$ accumulation in $\mathrm{MCI}$ patients suggesting two patterns of accumulation, i.e., A-pattern and B-pattern. In A-pattern, the deposition of cerebral A $\beta$ is mainly observed in the frontal part of the brain. In B-pattern, 
on the other hand, the deposition is observed evenly throughout the whole brain. While the study reported that 11 out of 36 amyloid-positive aMCI patients had an A-pattern and 1 out of 3 amyloid-positive naMCI patients had an A-pattern, ${ }^{11}$ further investigation is still needed for larger naMCI sample.

Therefore, we aimed to examine the differences of amyloid positivity rate and accumulation pattern between aMCI and naMCI subtypes in MCI subjects including more naMCI individuals compared to previous studies.

\section{METHODS}

\section{Participants}

A total of $102 \mathrm{MCI}$ (34 naMCI and age-, sex-, and education matched $68 \mathrm{aMCI}$ ) patients were recruited from the pool of older adults who visited the Dementia and Age-Associated Cognitive Decline Clinic of the Seoul National University Hospital (SNUH) to get outpatient clinical services. All participants met the current consensus criteria for MCI: 1) cognitive impairment confirmed by an informant, 2) objective cognitive impairment, 3) preserved global cognitive function, 4) independence in functional activities, and 5) not demented. ${ }^{13,14}$ aMCI and naMCI subjects met different criterion 2). For aMCI, the age-, sex-, and education-adjusted z-score for at least one of the four episodic memory tests (i.e., Word List Memory, Word List Recall, Word List Recognition, and Constructional Recall tests) in the Korean version of the Consortium to Establish a Registry for Alzheimer's disease (CERAD-K) neuropsychological battery ${ }^{15,16}$ was below -1.5 . In contrast, for naM$\mathrm{CI}$, the age-, sex-, and education-adjusted z-score for all the four episodic memory tests were identical to or above -1.5 and at least one of four non-memory tests (i.e., Boston Naming, Visuospatial Construction, and Sematic Fluency in the CERAD-K neuropsychological battery, and Stroop Color Word test) was below -1.5. All participants had an overall clinical dementia rating $(\mathrm{CDR})^{17}$ of 0.5 . Exclusion criteria for all subjects were any serious medical or neurological diseases present that could affect mental function, evidence of focal brain lesions on magnetic resonance imaging, the presence of severe behavioral or communication problems that would make a clinical examination difficult, and the absence of a reliable informant. Individuals with minor physical abnormalities (e.g., diabetes with no serious complications, essential hypertension, and mild hearing loss) were included. This study was approved by the Institutional Review Boards of the SNUH (IRB No. 1905-110-1035).

\section{Clinical and neuropsychological assessments}

All participants underwent comprehensive clinical and neuropsychological evaluation based on the CERAD-K assessment packet. ${ }^{15,16}$ Clinical diagnosis and CDR rating was made through consensus case conference in which more than four psychiatrists with expertise for dementia research participated. The presence or absence of six cerebrovascular risk factors, including stroke, diabetes, dyslipidemia, transient ischemic attack, hypertension, and coronary artery disease, was systematically assessed from the subjects and subjects' histories provided by the informant as well as a review of pertinent medical records. To calculate an overall measure of cerebrovascular burden, we created a composite score, i.e., the vascular risk score (VRS), which was the sum of the factors present, ranging from 0 to 6 and reported as a percentage. ${ }^{18}$ Apolipoprotein E (APOE) genotyping was implemented for subset of participants $(n=81)$.

\section{Assessment for amyloid positivity and accumulation pattern}

All participant received $\left[{ }^{18} \mathrm{~F}\right]$ florbetaben positron emission tomography (PET) scan. PET scans were performed using the ECAT EXACT47scanner (Siemens-CTI; Knoxville, TN, USA). The participants were classified as either $A \beta$ positive or negative according to the Brain Amyloid Plaque Load (BAPL) score. ${ }^{19}$ The BAPL is a three-grade scoring system, which is determined by visual assessment of the degree of $A \beta$ accumulation. If the BAPL score is 2 or 3 points in at least one of the four regions of Interest (ROIs), i.e., the lateral temporal lobes, frontal lobes, posterior cingulate/precuneus and parietal lobes, the patient is classified to $A \beta$ positive. A trained psychiatric resident (SHL) rated the BAPL score for PET scan of every participant. In order to verify the reliability of the rating, the agreement between ratings of the rater (SHL) and clinical ratings provided by nuclear medicine physicians at the SNUH was evaluated by calculating kappa value. More than substantial agreement was observed: Kappa value was 0.729 for aMCI, 1.000 for naMCI, and 0.822 for overall MCI cases. For $\mathrm{A} \beta$ positive participants, $A \beta$ accumulation pattern was also determined by visual inspection by the same rater (SHL). The accumulation pattern was classified into A-pattern and B-pattern according to the guideline described in the previous report. ${ }^{11}$ A-pattern has predominant amyloid retention in the frontal, anterior cingulate, lateral temporal, and basal ganglia, while B-pattern shows generalized amyloid retention over the entire cerebral cortex (Figure 1).

\section{Statistical analysis}

All statistical analyses were conducted using SPSS (version 24, IBM Corp., Armonk, NY, USA). For the comparison between aMCI and naMCI, Student's t-test or chi-square test was used depending on the type of variables. The threshold for statistical significance was $\mathrm{p}<0.05$ for all the analyses. 

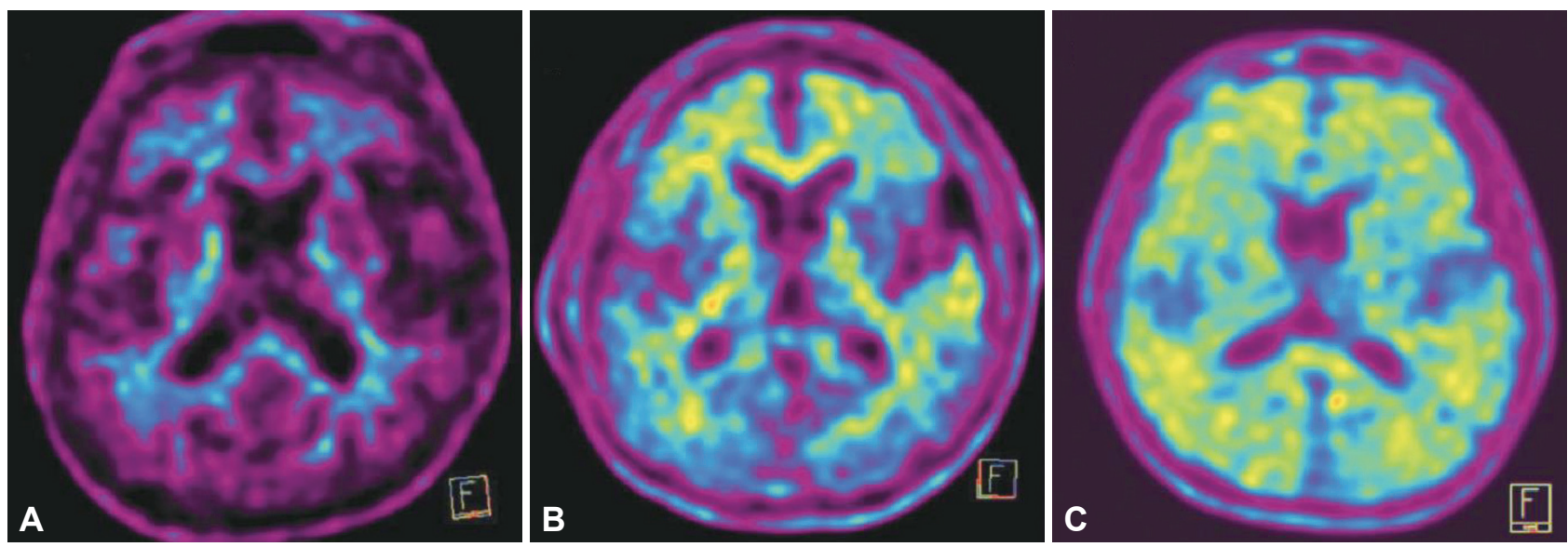

Figure 1. Cerebral beta-amyloid protein $(A \beta)$ accumulation patterns of $\left[{ }^{18} \mathrm{~F}\right]$ florbetaben $P E T$ image. $A: A \beta$ negative. $B$ : Predominant $A \beta$ retention in frontal, anterior cingulate, lateral temporal, and basal ganglia (A-pattern). C: Generalized A $\beta$ retention (B-pattern).

\section{RESULTS}

\section{Demographic and clinical characteristics}

Table 1 shows the demographic and clinical characteristics of the participants by MCI subtype. Since the participants of aMCI and naMCI were matched by age, sex and years of education, there was no significant difference in the demographic factors. The two MCI subtypes also did not differ in regard of age of onset, duration of illness, APOE $\varepsilon 4$ carrier state, and VRS. The mean CDR sum of box (SOB) score of aMCI was significantly higher than that of naMCI. As expected, z-scores of four episodic memory tests in the CERAD-K neuropsychological batter were significantly lower in aMCI than in naMCI.

\section{$A \beta$ positivity rate}

The $\mathrm{A} \beta$ positivity rate of aMCI and naMCI was $64.7 \%$ and $26.5 \%$, respectively (Table 2, Figure 2). The rate of naMCI was significantly lower than that of aMCI.

\section{$\mathrm{A} \beta$ accumulation pattern}

There was no significant difference in the proportion of amyloid accumulation patterns between $A \beta$ positive $\mathrm{aMCI}$ and $\mathrm{A} \beta$ positive naMCI (Table 3, Figure 2).

\section{DISCUSSION}

In the present study, cerebral $A \beta$ positivity rate was significantly higher in aMCI compared with naMCI. There was no difference in the proportion of $A \beta$ accumulation patterns between aMCI and naMCI cases with amyloid positivity.

The $\mathrm{A} \beta$ positivity rate for aMCI $(64.7 \%)$ observed in this study was very similar to the rates reported in previous studies, which ranged from $57.9 \%$ to $67.9 \%{ }^{9-12}$ In regard of the $A \beta$
Table 1. Demographic, clinical and neuropsychological characteristics of mild cognitive impairment subtypes

\begin{tabular}{|c|c|c|c|}
\hline Variables & $\begin{array}{c}\mathrm{aMCI} \\
(\mathrm{N}=68)\end{array}$ & $\begin{array}{l}\text { naMCI } \\
(\mathrm{N}=34)\end{array}$ & p-value \\
\hline \multicolumn{4}{|c|}{ Demographic and clinical characteristics } \\
\hline Age (y) & $74.6 \pm 6.5$ & $74.3 \pm 6.9$ & 0.801 \\
\hline Female, N (\%) & $58(85.3)$ & $29(85.3)$ & 1.000 \\
\hline Education (y) & $9.37 \pm 4.97$ & $8.8 \pm 4.71$ & 0.597 \\
\hline Age of onset (y) & $71.7 \pm 7.5$ & $71.5 \pm 7.2$ & 0.910 \\
\hline Duration of illness (y) & $2.99 \pm 2.31$ & $2.76 \pm 1.58$ & 0.617 \\
\hline APOE4 carrier, $\mathrm{N}(\%)^{*}$ & $22(40.0)$ & $7(26.9)$ & 0.252 \\
\hline CDR SOB & $1.71 \pm 0.66$ & $1.43 \pm 0.64$ & 0.044 \\
\hline VRS total & $1.31 \pm 1.08$ & $1.65 \pm 1.10$ & 0.142 \\
\hline \multicolumn{4}{|c|}{ Neuropsychological tests (z-score) } \\
\hline MMSE & $-1.12 \pm 0.98$ & $-0.42 \pm 0.85$ & 0.001 \\
\hline Semantic fluency & $-0.69 \pm 0.96$ & $-0.76 \pm 0.84$ & 0.721 \\
\hline Boston naming & $-0.11 \pm 1.34$ & $-0.26 \pm 1.23$ & 0.574 \\
\hline Word list memory & $-1.09 \pm 0.94$ & $-0.25 \pm 0.57$ & $<0.001$ \\
\hline Word list recall & $-1.70 \pm 0.92$ & $-0.23 \pm 0.76$ & $<0.001$ \\
\hline Word list recognition & $-1.36 \pm 1.82$ & $-0.15 \pm 0.76$ & $<0.001$ \\
\hline Constructional praxis & $-0.24 \pm 1.14$ & $-0.25 \pm 1.05$ & 0.971 \\
\hline Constructional recall & $-1.25 \pm 0.84$ & $-0.32 \pm 0.76$ & $<0.001$ \\
\hline Stroop color-word & $-0.92 \pm 1.09$ & $-1.26 \pm 0.95$ & 0.115 \\
\hline
\end{tabular}

Data are presented as means \pm SD for continuous variables and as $\mathrm{N}$ (\%) for categorical variables with $\mathrm{p}$-values for comparison between aMCI and naMCI. Continuous variables were analyzed through Student's t-test and categorical variables were analyzed through chisquare test. *number of participants who underwent APOE genotyping were 81 (aMCI 55, naMCI 26). aMCI: amnestic mild cognitive impairment, naMCI: non-amnestic mild cognitive impairment, APOE4: apolipoprotein E ع4, CDR-SOB: Clinical Dementia Rating sum of box, VRS: vascular risk score, MMSE: Mini-Mental State Examination 
Table 2. Comparison of $A \beta$ positivity rate between mild cognitive impairment subtypes

\begin{tabular}{lccc}
\hline & aMCI $(\mathrm{N}=68)$ & naMCI $(\mathrm{N}=34)$ & p-value \\
\hline A $\beta$ positive & $44(64.7)$ & $9(26.5)$ & $<0.001$ \\
A $\beta$ negative & $24(35.3)$ & $25(73.5)$ & \\
\hline
\end{tabular}

Data are presented as N (\%). P-value for comparison of aMCI and naMCI with chi-square test. A $\beta$ : beta-amyloid protein, aMCI: amnestic mild cognitive impairment, naMCI: non-amnestic mild cognitive impairment

Table 3. Comparison of the proportion of $A \beta$ accumulation patterns between $\mathrm{A} \beta$ positive $\mathrm{aMCl}$ and naMCl

\begin{tabular}{lccc}
\hline & aMCI $(\mathrm{N}=44)$ & naMCI $(\mathrm{N}=9)$ & p-value \\
\hline A-pattern & $20(45.5)$ & $3(33.3)$ & 0.504 \\
B-pattern & $24(54.5)$ & $6(66.7)$ & \\
\hline
\end{tabular}

Data are presented as N (\%). P-value for comparison of aMCI and naMCI with chi-square test. $A \beta$ : beta-amyloid protein, aMCI: amnestic mild cognitive impairment, naMCI: non-amnestic mild cognitive impairment

positivity rate for naMCI, our result obtained from relatively larger sample [9 of $34(26.5 \%)$ ] was slightly higher than the rates from two previous studies with smaller sample [ 3 of 17 $(17.6 \%)^{10}$ and 3 of $16(18.8 \%)^{11}$, while lower than those from other two small studies [6 of $18(33.3 \%)^{9}$ and 3 of $7(42.9 \%)^{12}$ ]. As various factors including demographic variables, study settings, PET ligands used to measure cerebral $A \beta$, and the definition or threshold for $A \beta$ positivity may influence on the rate, so it is not easy to directly compare it between the studies. Nevertheless, as shown in our study, the $A \beta$ positivity rate of aMCI was higher than that of naMCI across all the studies, consistent with clinical observation that higher proportion of individuals progressed to $\mathrm{AD}$ dementia in $\mathrm{MCI}$ than in naMCI. ${ }^{6-8}$

We also compared the proportion of $A \beta$ accumulation patterns between aMCI and naMCI and did not find any significant difference. This observation indicates that regional distribution pattern of cerebral $A \beta$ accumulation is not associated with the difference of impaired cognitive domain in MCI. A previous study also reported similar result although the study included only three $\mathrm{A} \beta$ positive naMCI subjects. ${ }^{11}$

Although previous clinical studies suggested that naMCI individuals are more related with vascular dementia than aMCI ones, ${ }^{6,20}$ we did not find any difference of VRS between the two subtypes. The lack of significant difference might be attributable to the small sample size or measurement method for vascular burden. When compared for the proportion of subjects who have at least one vascular risk factor, there were significant difference between aMCI and naMCI $(70.6 \%$ for aMCI vs. $88.2 \%$ for naMCI, $\mathrm{p}=0.048$ ).

Although the present study has a strength in that relatively large number of naMCI patients were included, a couple of limitations should also be mentioned. First, as the MCI sub-

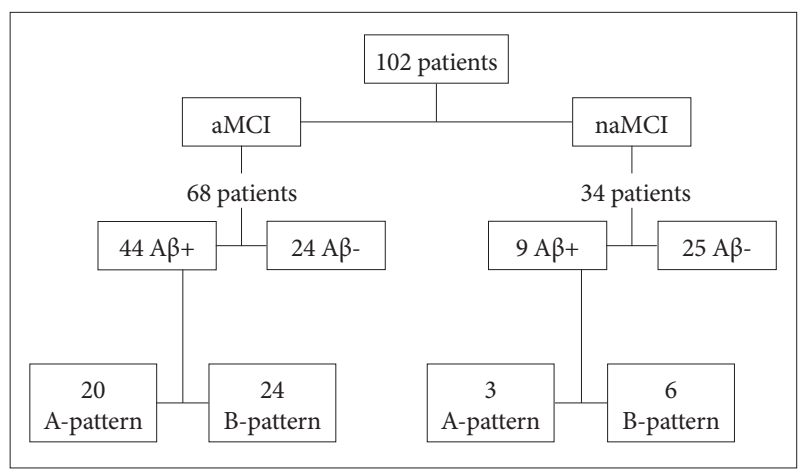

Figure 2. Distribution of cerebral beta-amyloid protein $(A \beta)$ positive and negative cases in amnestic mild cognitive impairment (aMCl) and non-amnestic mild cognitive impairment (naMCI), and distribution of A-Pattern and B-Pattern accumulation in $\mathrm{A} \beta$ positive $\mathrm{aMCl}$ and naMCl.

jects were recruited from a dementia clinic in tertiary hospital, there may be some limitation to generalize the results to community population. Second, this study did not evaluate other pathologies frequently found in brain of older adults, such as cerebral tau, alpha synuclein, TDP-43, and vascular pathologies. To get more understandings for the causative neuropathologies underlying MCI subtypes, future studies using other brain imaging modalities or post-mortem brains are necessary.

In conclusion, the current findings suggest that MCI subtypes based on impaired cognitive domains have a differential association with brain $\mathrm{A} \beta$ deposition, a core pathology of $\mathrm{AD}$. Amnestic subtype of $\mathrm{MCI}$ are more closely associated with cerebral $\mathrm{A} \beta$ deposition compared to non-amnestic subtype. In contrast, the pattern of amyloid deposition does not appear to have any difference between the subtypes.

\section{Acknowledgments}

This study was supported by a grant from Ministry of Science and ICT, Republic of Korea (Grant No. NRF-2014M3C7A1046042) and a grant of the Korea Health Technology R\&D Project through the Korea Health Industry Development Institute (KHIDI), funded by the Ministry of Health \& Welfare, Republic of Korea (Grant No: HI18C0630 \& HI19C0149).

\section{Conflicts of Interest}

The authors have no potential conflicts of interest to disclose.

\section{Author Contributions}

Conceptualization: Dong Young Lee, Jun Ho Lee, Sun Hyung Lee. Data curation: Jun Ho Lee, Sun Hyung Lee. Formal analysis: Sun Hyung Lee, Jun Ho Lee. Funding acquisition: Dong Young Lee. Investigation: Dong Young Lee, Jun Ho Lee, Min Soo Byun, Dahyun Yi, Gijung Jung, Jee Eun Park. Methodology: Dong Young Lee, Jun Ho Lee, Min Soo Byun, Dahyun Yi. Project administration: Dong Young Lee, Jun Ho Lee, Min Soo Byun, Dahyun Yi, Gijung Jung, Jee Eun Park. Resources: Dong Young Lee, Jun Ho Lee, Min Soo Byun. Supervision: Dong Young Lee, Jun Ho Lee. Validation: Dong Young Lee, Jun Ho Lee. Visualization: Sun Hyung Lee. Writing-original draft: Sun Hyung Lee. Writing_review \& editing: Dong Young Lee, Jun Ho Lee, Min Soo Byun, Dahyun Yi, Gijung Jung, Jee Eun Park. 


\section{ORCID iDs}

Sun Hyung Lee

Jun Ho Lee

Min Soo Byun

Dahyun Yi

Gijung Jung

Jee Eun Park

Dong Young Lee https://orcid.org/0000-0001-8559-8117 https://orcid.org/0000-0002-1809-5154 https://orcid.org/0000-0003-3159-4510 https://orcid.org/0000-0002-0860-0946 https://orcid.org/0000-0001-5952-3373 https://orcid.org/0000-0001-6097-3275 https://orcid.org/0000-0001-8976-8320

\section{REFERENCES}

1. Winblad B, Palmer K, Kivipelto M, Jelic V, Fratiglioni L, Wahlund LO, et al. Mild cognitive impairment-beyond controversies, towards a consensus: report of the International Working Group on Mild Cognitive Impairment. J Intern Med 2004;256:240-246.

2. Petersen RC, Morris JC. Mild cognitive impairment as a clinical entity and treatment target. Arch Neurol 2005;62:1160-1163.

3. Gauthier S, Reisberg B, Zaudig M, Petersen RC, Ritchie K, Broich K, et al. Mild cognitive impairment. Lancet 2006;367:1262-1270.

4. Petersen RC. Mild cognitive impairment. Continuum (Minneap Minn) 2016;22:404-418.

5. Roberts R, Knopman DS. Classification and epidemiology of MCI. Clin Geriatr Med 2013;29:753-772.

6. Nordlund A, Rolstad S, Klang O, Edman A, Hansen S, Wallin A. Two-year outcome of MCI subtypes and aetiologies in the Goteborg MCI study. J Neurol Neurosurg Psychiatry 2010;81:541-546.

7. Mitchell J, Arnold R, Dawson K, Nestor PJ, Hodges J. Outcome in subgroups of mild cognitive impairment (MCI) is highly predictable using a simple algorithm. Neurology 2009;256:1500-1509.

8. Rasquin S, Lodder J, Visser P, Lousberg R, Verhey F. Predictive accuracy of MCI subtypes for Alzheimer's disease and vascular dementia in subjects with mild cognitive impairment: a 2-year follow-up study. Dement Geriatr Cogn Disord 2005;19:113-119.

9. Patricio CM, Gabriela C, Julieta RM, Marcos FS, Federico N, Griselda $\mathrm{R}$, et al. Concordance between 11C-PIB-PET and clinical diagnosis in a memory clinic. Am J Alzheimers Dis Other Demen 2015;30:599-606.

10. Jiménez-Bonilla JF, Banzo I, De Arcocha-Torres M, Quirce R, MartínezRodríguez I, Lavado-Pérez C, et al. Diagnostic role of 11C-Pittsburgh compound $\mathrm{B}$ retention patterns and glucose metabolism by fluorine-18fluorodeoxyglucose PET/CT in amnestic and nonamnestic mild cognitive impairment patients. Nucl Med Commun 2016;37:1189-1196.

11. Banzo I, Jimenez-Bonilla JF, Martinez-Rodriguez I, Quirce R, de Arcocha-Torres M, Bravo-Ferrer Z, et al. Patterns of 11C-PIB cerebral retention in mild cognitive impairment patients. Rev Esp Med Nucl Imagen Mol 2016;35:171-174.

12. Wolk DA, Price JC, Saxton JA, Snitz BE, James JA, Lopez OL, et al. Amyloid imaging in mild cognitive impairment subtypes. Ann Neurol 2009; 65:557-568.

13. Petersen RC. Mild cognitive impairment as a diagnostic entity. J Intern Med 2004;256:183-194.

14. Petersen RC, Doody R, Kurz A, Mohs RC, Morris JC, Rabins PV, et al. Current concepts in mild cognitive impairment. Arch Neurol 2001;58: 1985-1992.

15. Lee DY, Lee KU, Lee JH, Kim KW, Jhoo JH, Kim SY, et al. A normative study of the CERAD neuropsychological assessment battery in the Korean elderly. J Int Neuropsychol Soc 2004;10:72-81.

16. Lee JH, Lee KU, Lee DY, Kim KW, Jhoo JH, Kim JH, et al. Development of the Korean version of the Consortium to Establish a Registry for Alzheimer's Disease Assessment Packet (CERAD-K) clinical and neuropsychological assessment batteries. J Gerontol B Psychol Sci Soc Sci 2002;57:47-53.

17. Morris JC. The Clinical Dementia Rating (CDR): current version and scoring rules. Neurology 1991;41:1588-1592.

18. DeCarli C, Mungas D, Harvey D, Reed B, Weiner M, Chui H, et al. Memory impairment, but not cerebrovascular disease, predicts progression of MCI to dementia. Neurology 2004;63:220-227.

19. Sabri O, Seibyl J, Rowe C, Barthel H. Beta-amyloid imaging with florbetaben. Clin Transl Imag 2015;3:13-26.

20. Busse A, Hensel A, Gühne U, Angermeyer M, Riedel-Heller S. Mild cognitive impairment: long-term course of four clinical subtypes. Neurology 2006;67:2176-2185. 